\title{
TESIS DIDÁCTICAS
}

\begin{abstract}
CONOCIMIENTO Y ACCIÓN EN LA ENSENANZA DE CONCEPTOS MATEMATICOS Y LA RESOLU. CIÓN DE PROBLEMAS, DE PROFESORES DE EGB CON EX. PERIENCIA, ESPECIALISTAS EN MATEMÁTICAS Y ESTUDIANTES PARA PROFESORES
\end{abstract}

\section{Tesis Doctoral}

Autor: Lorenzo Blanco Nieto. Profesor Titular de Didáctica de las Matemáticas. Miembro del Departamento de Didáctica de las Ciencias Experimentales y de las Matemáticas de la Universidad de Extremadura.

Director: Luis $M$. Villar Angulo.

Lugar: Departamento de Didáctica y Organización escolar de la Universidad de Sevilla.

Fecha: Septiembre de 1990.

EI trabajo de investigación describe y justifica las diferencias que se establecen en la enseñanza interactiva entre profesores de EGB con experiencia y alumnos de la Escuela de Formacion de Profesorado de EGB, en la especialidad de Ciencias, asumiendo que los resultados de esta experiencia pueden aportar elementos nuevos para ser considerados en los proyectos de reforma de la Formación Inicial y Permanente deI profesorado, en los niveles de la enseñanza primaria y secundaria obligatoria.

El análisis de las decisiones, el estudio de las diversas situaciones y factores que inciden en el desarrollo de la clase y en las prấcticas de enseñanza de los alumnos de Magisterio, la consideración de los pensamientos y preocupaciones de los profesores durante la enseñanza constituye una línea de investigación que ha ayudado a delimitar, de manera más cficaz, nuevas formas de comprender $y$ describir la enseñanza de las Matemáticas.

Dentro del marco de investigación que representa el paradigma del pensamiento de los profesores, se sitúan diversos estudios que tratan de la enserianza interactiva. Es, en este marco, en el que se ha desarrollado el trabajo para conocer los nexos de unión entre el pensamiento y la acción, aceptando que el mismo ayuda a descubrir e interpretar la práctica docente, en los niveles señalados.

Por io tanto, la investigación se enmarca dentro de dos aspectos complementarios. Por un lado, la descripción y comprensión de la actividad docente de los maestros con experiencia, de cara a establecer ciertas pautas para la Didáctica de las Matemáticas y la formación permanente. De otra parte, queda señalado un camino de estudio e investigación sobre los comportamientos y pensamientos de los estudiantes para profesores que debe servirnos de punto de partida de renovación de los actuales currículos para su formación, es decir, para la formación inicial tanto de enseñanza primaria como secundaria.

Dentro de este marco se ha establecido con claridad el objeto de la investigación que aparece en el primer capítulo de la memoria presentada:

«Analizar la enseñanza interactiva de profesores de EGB, con experiencia en la enseñanza de las Matemáticas, y de estudiantes para profesores, durante las prácticas do enseñanza, para contrastar el pensamitento y la acción en relación con la enseñanza de las Matemáticas, que nos ayude a comprender el conocimiento práctico personal de los profeso. res expertos y noveles" (p. 29).

En el capítulo II se hace un recorrido sobre aquellos acontecimientos más sig nificativos a partir de los cuales las diferentes y sucesivas concepciones sobre la enseñanza da las Matemáticas han basado su evoluaion. Se hace referencia al «Movimiento en favor de la Matemática modema» que surgió en la deccada de los 60, y que provocó una corriente contraria en el «Movimiento para volver a lo básicon, en la década siguiente. Se recogen las principale:s ideas del «Movimientoen favor de la Resolución de Problemas», para terminat citando las nuevas orientaciones establecidas para «Las Matemáticas esenciales para el Siglo XXI».

Se exponen referencias a trabajos concretos que, en una línea semejante a la establecida en esta tesis, han servido de apoyo para plantear y reflexionar sobre las objetivos propuestos, así como sobre la metodología desartollada. A este respecto, se han considerado trabajos de investigación acerca del conocimiento pedagógico de Matemáticas, tanto de profesores expertos y noveles, considerados separadamente o para establecer una comparación entre ellos.

En el Capítulo III se hace una exposición de la metodología cualitativa desarrollada dando las pautas concretas que han servido para poder entrar, conocer y analizar el mundo de los participantes. Las observaciones de clase, a través de las grabaciones en audio y vídeo, y las entrevistas a los participantes, antes y despurs de las observaciones, han sido las técnicas básicas que han proporcionado los datos base de la investigación.

El análisis realizado muestra los elementos más significatives sobre el conocimiento práctico de los profesores, que han sido contrastados con las aportaciones que se establecen desde perspectivas teóricas de la Didáctica de las Matemáticas, estableciéndose un puente entre las nuevas propuestas curriculares y la actividad profesional de los profesores.

Los resultados obtenidos llevan a la consideración de conclusiones sobre los conocimientos y acción desarrolladas por los informantes en Ia investigación, que sugieren implicaciones curriculares a tener en cuenta, tanto en cursos de formación permanente como en los futuros diseños para la formación inicial.

Los puntos señalados en el Capítulo $\mathrm{V}$ deben implicar la modificación de algunos parámetros considerados en la formación de los profesores de Matemáticas tanto en los aspectos de contenidos desarrollados en ios programas de Didúctica de las Matemáticas, como en la metodología desarrollada en estas mismas clases.

Por último, los resultados y conclusiones generados en este trabajo aportan elementos claros de crítica a los actuales sistemas de prácticas de los estudiantes de Magisterio, sugiriéndose en las implicaciones algunas líneas que marcarían su renovación. 
LA VELOCIDAD: INTRODUCCIÓN AL, CONCEPTO DE DERIVADA

\section{Tesis doctoral}

Autora: Carmen Azcárate Giménez. Departament de Didactica de la Matematica $i$ de les Ciències Experimentals de la $U A B$.

Director: Dr. David Johnson (King's College of London University).

Lugar: Bellaterra. Facultat de Ciències (Físiques) de la UAB

Fecha: Diciembre de 1990.

\section{Resumen}

Fl objetivo de esta investigacion ha sido el de analizar el proceso de aprendizaje, en la asignatura de Matemáticas, de los alumnos de tres clases de Segundo de BUP que han seguido el material curricular del Grup Zero Introducció a les derivades (1980, revisado en 1987); los profesores de las tres clases eran micmbros del Grup Zero.

El itinerario didáctico de esta introducción al concepto de derivada de una función en un punto está pensado para alumnos que no han estudiado previamente los conceptos ni de límite ni de continuidad. El punto clave de este estudio radica en el concepto de recta tangente a una gráfica en un punto, concepto que, a diferencia del de límite de una función, tiene un soporte graffico que favorece un enfoque intuitivo. Por otra parte, en este material curricular se introduce el conccpto de derivada de una función en estrecha relación con el concepto de velocidad instantánea que es el ejemplo más sencillo de derivada con nombre propio que está asociado a experiencias extraescolares cotidianas (lectura de un velocímetro).

La investigación sc ha centrado en el estudio y en el seguimiento de los esquemas conceptuales de los alumnos referentes a tres conceptos fundamentales relacionados con la noción de derivada: el concepto de pendiente de una recta, el concepto de velocidad instantánea de un movimiento variado y el concepto de tasa instantánea de variación de una función (o valor de la función derivada en un punto). Se ha completado, además, esta investigación con el análisis de los procedimientos utilizados por los alumnos.

Este trabajo se inscribe en el marco de las investigaciones educativas basadas en datos cualitativos, expresados en forma verbal y obtenidos a partir de entrevistas $y$ de cuestionarios escritos de preguntas abiertas. De acuerdo con ello, se ha realizado una recogida de datos secuenciada: uncuestionario escrito y una entrevista previos a la fase de aprendizaje, una entrevișta previa a la lección de formalización del concepto de tasa instantánea de variación. y una entrevista y un cuestionario escrito posteriores a la fase de aprendizaje. En cuanto a la muestra, ha consistido en las tres clases completas (111 alumnos) para los dos cuestionarios escritos y en 6 alumnos por clase (siempre los mismos) para cada una de las tres entrevistas.

El análisis se ha centrado en el estudio y en la clasificación de las respuestas de los alumnos en los aspectos conceptuales, lo que ha permitido establecer lo que se ha denom nado perfiles de los alumnos, característicos en cada momento de sus esquemas conceptuales de cada uno de los tres conceptos estudiados.

En cuanto al concepto de pendiente de una recta se han identificado tres perfiles que hemos llamado "geométrico», uope. rativo" $y$ "funcional»:

- El perfil «geométrico» que corresponde al aspecto menos abastracto, más sim. ple e intuitivo y más reforzado por el significado del término en el lenguaje cotidiano, caracteriza a los alumnos cuyo esquema conceptual consiste simplemente en imágenes graf́ficas y cuya descripción utiliza elementos propios del lenguaje geométrico, tanto descriptivo como analítico.

- El perfil «operativo», que corresponde al aspecto más escolar en el sentido del quehacer habitual de los ejercicios de clase en los que se calcula la pendiente a partir de una ecuación, caracteriza a los alumnos cuyo esquema conceptual tiene una fijación numérica de manera que, en una fórmula del tipo

$$
y=a \cdot x+b \text {, }
$$

asocian a la palabra «pendiente» el coeficiente «a» o la expresión polinómica «a. $x+b »$.

- El perfil «funcional», que está relacionado con el significado de pendiente como tanto por uniđad o variación de la función por unidad, es el que corresponde al aspecto menos intuitivo, más abstracto y más complejo, caracteriza a los alumnos cuyo esquema conceptual asocia a la palabra "pendiente» una relación de dependencia tineal entre los incrementos de las variables de la función, así comola imagen gráfica de una recta en la que destacan diches incrementos.

En cuanto a los conceptos de velocidad instantánea y cle tasa instantánea de variación de una función se han determinado tres tipos de esquemas conceptuales de los alumnos que corresponden a los perfiles que hemos llamado "primitivo», «aproximación» y «límite»:

- El perfil «primitivo» corresponde a alumnos que no han construido un es- quema conceptual específico de las nociones de velocidad instantánea o de tasa instantánea de variación de una función. Estos alumnos tienen un esquema conceptual de "velocidad en general» que responde a la formulación verbal: «la velocidad es igual al espacio partido por el tiempo", muy estable y coherente, que no distingue entre velocidad constante, velocidad media y velocidad en un punto. La principal dificultad de estos alumnos consiste en la confusión entre velocidad media desde el origen y velocidad instantánea en un punto.

- El perfil «aproximación» corresponde a alumnos que, en el caso del concepto de velocidad instantánea, han generalizado su esquema conceptual de la noción de velocidad media a la noción de velocidad media entre dos puntos próximos que les sirve ahora para describir de manera aproximada la velocidad en un punto dado y, en el caso de la descripción de la variación puntual de una función, han realizado una transposición a partir de su esquema conceptual de velocidad media, que ha dado lugar a un esquema conceptual de tasa media de variación. En ambos casos, los esquemas conceptuales son coherentes y sirven para resolver situaciones puntuales por aproximacion.

- El perfj! «límite» corresponde a alumnos que, durante la fase de aprendizaje, han construido unos esquemas conceptuales tanto de la noción de velocidad instantánea como de la noción de tasa instantanca de variación de una función en un punto, de mancra que interpretan, describen y representan situaciones de variación instantánea de una función dada por su gráfica.

El estudio de la evolución de los perfiles de los alumnos a lo largo de la fase de aprendizaje revela que ha habido un cambio y una evolución de progreso importante en los esquemas conceptuales de los alumnos, ya que se aprecia un desplazamiento desde un gran número de perfiles «primitivo» en la situación inicial hasta un gran número de perfiles «límite» en la situación final, pasando por una mayoría de perfiles «aproximación» en el iranscurso de la fase de aprendizaje.

Se ha observado que los alumnos tienden a utilizar un sóto lenguaje, sea verbal, simbólico o gráfico, y que les cuesta pasar de uno a otro, lo cual indica una cohesión frágil de su esquema conceptual revelador de la dificultad que existe en la asimilación de todos los aspectos de un concepto.

En el aspecto metodológico, se han utilizado las redes sistémicas como instrumento de análisis de tas respuestas de los aímnos a los cuestionarios escritos que han permitidoconsiderar simuitáneamente un gran número de respuestas variadas y prepararlas para su descripción, agru- 
pándolas según diversos criterios. Por otra parte, para estudiar la cvolución de los alumnos ha sido útil la representación gráfica en cuadros de doble entrada con una dimensión horizontal temporal y una dimensión vertical ordenada con Ios modelos de respuesta o con los perfiles de los alumnos.

OPTYQUE ÉLÉMENTAIRE EN CLASSE DE QUATRIËME:

RAISONS ET IMPACT SUR LES MAÎTRES D'UNE MAQUETTE D'ENSEIGNEMENT

\section{Diploma de Doctorat}

Autor: Wanda Kaminski

Director: Dr. L. Viennot

Lloc: Université Paris 7.

\section{Résumé}

Le présent travail prend pour point de départ les études exploratoires sur ies conceptions des élèves et des adultes en optique élémentaire. La première partie, centrée sur le thème de la chambre noire, presente une investigation des difficultés courantes, oì il apparaît que tes notions les plus fondamentales du domaine sont loin d'être évidentes, et suscitant des difficultés très semblables chez les élèves et les maîtres des classes de quatrième.

Au cours de I'investigation, des questions sont posées devant des montages expérimentaux représentant des situations légèrement modifiés par rapport à la chambre noire classique. Les activités proposées aux élèves et à un groupe de maîtres sont sollicités en tant que «juges». Les enseignants-juges vérifient d'abord les prévisions correctes en manipulart les mêmes montages expérimentaux qui ont servi auparavant pour interroger les elèves et le premier groupe de maîtres. Ils examinent ensujte toutes les réponses recueillies pour décider, selon leurs propres critères, si elles sont «acceptabies». L'analyse de ces réponses déclarées acceptables donne des indications sur les objectifs d'enseignement. D'une part les schémas «acceptables» sont bien compatibles avec la propagation rectiligne, mais non avec la propagation isotrope de la lumière, d'autre part le type de schéma «appris en classe» n'est pas intégré dans un raisonnement cohérent. Quant aux prévisions «acceptées», elles révèlent un «flou conceptuel» associé principalement à la notion de lumière elie-même, au fait que celie-ci soit invisible «de profil» et au rôle de l'oeil dans la vision. Toutes ces difficultés ne semblent guère perçues par les profess:urs-juges.

A partir de ces résultats, I'auteur définit une liste d'objectifs conceptuels pour un enseignement qui forme un ensemble cohérert sur une durée d'une trentaine d'heures.

La spécification de cet enseignement est faite dans un second chapitre où sont précisés, sous la forme de cinq séquences, objectifs, interventions du maitre, manipuations et questions posées, difficultés à prévoir et siłuations susceptibles d'enrichir la discussion avec les formés. L'activité de ceux-ci est constamment sollicitée, notamment par des exercices du type «prévision/confrontation de la lumière et à la synthèsc des couleurs; la deuxièmı - à la propagation rectiligne et isotrope de la lumière; la troisième - a la formation des images par des lentilles minces zonvergentes; ja quatrième - à l'analyse de ce qu'est une "lumière visualisée"; et la dernière- à un thème présenté en tant que synthèse: la cham. bre noirt. L'ordre de ces séquences, ainsi que l'ordre des différents épisodes à l'intérieur d'une séquence, suit une logi-

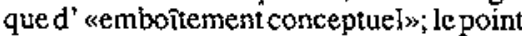
de départ est l' «état conceptuel» de l'élève, et les activités proposées au cours d'un episode ont pour but l'acquisition d'un ou de deux concepts-《cibles» qui, au cours des épisodes suivants, servent comme "concepts-outils» pour aider à atteindre les nouvelles «cibles» conceptuelles

La troisième partie fournit des éléments d'une double évaluation; d'une part celle des acquisitions conceptuelles des maîtres ayant eux-mêmes reçu l'enseignement décrit plus haut, d'autre part, celle du degré et tes raisons de leur adhésion au projet d'en reprendre à leur compte des éléments vis+a-vis de leurs propres élèves. Les informations recueillies de ce double point de vue, notamment les importantes restructurations observées chez les stagiaires dans l'appréciation des difficultês du domaine, fournissent des éléments précis en vue d'une expérimentation directe sur les elèves.
FORMACIÓ DE CONCEPTES AL VOLTANT DELS CANVIS D'ESTAT D'AGREGACIÓ DE LA MATERIA. CAS PARTICULAR: FIS EQUILIBRIS LIQQUID-VAPOR

\section{Tesis doctoral}

Autor: Josep Rafel Montala. I.B. Besòs Barcelona.

Directors: Dr. Claudi Mans. Facultat de Química. Universitat de Barcelona. Dr. Paul Black. King's College. University of London.

Lloc: Universitat de Barcelona.

Data: 1990.

\section{Resum}

L'objectiu d'aquesta primera tesi en Di* dàctica de la Química presentada a la Facultat de Química de la Universitat de Barcelona fou la investigació de preconceptes en els nostres atumnes de batxillerat, sobre els canvis d'estat d'agregació de la matèria i l'obtenció d'algunes directrius per a la nostra posterior actuació a les classes de Física i Química.

S'escollí el tema dels canvis d'estat, atès cl seu interès cientiffic i cultural que fa que sigui inclòs tant al currículum de Ciències de l'escola primària, com al de Física i Química de Secundaria, des de $7 \mathrm{èd} E \mathrm{~d} B$ fins al COU. Concretament els equilibris líquid-vapor i cspecialment el cas concret del cicle de l'aigua és un dels fenòmens naturals esencials més importants com ja indicava Bar, i per la seva vinculació a la vida quotidiana és motiu d'interaccions culturais i lingitístiques ja des de la primera infància (ben abans dels quatre o cinc anys) amb uncs grans possibilitats de condicionar l'ensenyament dels conceptes científics que es rebran a l'escola o a l'institut).

La historia de les recerques sobre idees dels alumnes realitzades majorment des del constructivisme amb prou feines arriba als 20 anys, a part d'algunes investigacions axillades anteriors, fonamentalment les de Piaget i dels psicòlegs de l'Escola de Ginebra, i descomptant, és clar, els escrits de 1710 de Giambattista Vico de principi del segle XVIII.

Els treballs de Driver, Easley, Fensham o Viennot, entre altres, van obrir un nou camí en el camp de la recerca en Didàctica de les Ciències. Un dels focus d'aquest tipus de recerca ha estat el King's College dc Londres on alguns dels seus professors han participat en projectes S.P.A.C.E., etc. $i$ han dirigit nombroses recerques en Didàctica de les Ciències a estudiants de tot el món.

D'altra banda, la tradició en recerca sobre la Didàctica de les Ciencies fins als anys 80 era gairebé inexistent a Catalu- 
nya, descomptant algunes aportacions aillades dels anys 30 . Igualment les eines de professionalització com a professors de Secundària dels Enginyers y dels Hicenciats en Biologia, Geologia, Física, Química o Matematiques han estat nul.les, durant molts anys.

Amb aquests precedents, aquesta recerca s'inicià el 1985, amb una beca del Departament d'Ensenyament de la Generalitat de Catalunya per a tesis doctorals en Didàctica de les Ciències, que incloïa la col.laboració del King's College amb les Universitats catalanes.

Es realitzà un pre-test (103 alumnes) per a andlisis preliminars $i$ un test final ( 549 alumnes) amb els quals corroborar les hipotesis de treball. Aquests tests constaven de quiestions d'elecció múltiple i d'obertes. Per a l'anàlisi d'aquestes (amb xarxes sistemiques $\mathrm{i}$ anotant les frases peculiars), s'optà per una reducció de la mostra (156 alumnes). Tambés'efectuaren algunes entrevistes personals. El tractament de dades es realitzà amb el paquet SPSSx. Fonamentalment s'han utilitzat taules de contingència $\mathrm{i}$ el mètode LOGIT per a estudiar l'associacio o la dependència de les respostes respecte de variables de disseny del qüestionari $i$ d'altres variables socio-economiques $\mathrm{i}$ culturals.

Les principals conclusions d'aquesta recerca són:

- La certesa amb què els alumnes manifesten respondre a cada pregunta és independent de la bondat de la resposta. Aquesta conclusió és en la mateixa línia a la qual arriben altres investigadors, com Carrascosa i Gil, cosa que relacionen amb les bases per les quals les idees prèvies resulten tan persistents al llarg del temps, com ells mateixos, i Giordan entre altres, ens han demostrat.

- Les respostes obertes dels alumnes es poden classificat en categories que re- suiten coherents. Les principals categories assigrades a les frases són:

A) Correctes, de tipus vàlid, cientiftuques i enteses correctament.

B) Gairebé correctes lievat que són imprecises o que s'han entès malament.

F) Inadequiades que comprenen les del tipus $\mathrm{C}$ i D, que eren les que s'indiquen a continuacio:

C) Científiques inadequades (intents falitis d'explicacions científiques).

D) Respostes en llenguatge quotidia.

E) Sense utilitat o en blanc.

- Com a conseqüència, també s'han pogut elaborar diversos perfils d'alumne. en funció de les seves respostes obertes. Són bàsicament els següents:

- Alumnes avançats o científics.

- Alumnes coherents, encara que escas. sament científics.

- Alumnes singulars, categoria que comprèn la dels pseudo-científics i la dels quotidians.

- Grup irrellevant d'alumnes, sense utilitat per la seva manca de respostes utilitzables.

- Aquests perfils presenten associacions significatives amb:

- les categories assignades a les seves respostes,

- el rendiment obtingut o puntuacio global del test fínal si considerem les qứestions d'eleccio múltiple $i$ les qüestions obertes en conjunt.

- La coherència d'aquests perfits també s'ha pogut demostrar a la test.

- El rendiment dels estudiants en el test final, considerant les qüestions d'elecció múltiple i les quiestions obertes en conjunt, presenta associacions globals amb
Ia distribucio en 'Perfils' $\mathrm{i}$ amb l'entorn sòcio-cultural en guè viuen aquells.

No obstant, a l'unic perfjl que apareix independència respecte algún dels factors sòcio-eulturals dominants, és al perfit dels avançats. Es a dir per als alumnes del perfil més avançat la influència del seu 'entorn' no és tan determinant com per o les dels altres perfils.

- Alguns aspectes que sembla que bagin de tenir una mateixa dificultat que altres que hi guarden relacio, com és el cas de l'evaporació i la condensacio, resulta que són compresos de maneres ben diferents pels alumnes, tal con hem comprovat després d'examinar 30 qüestions sobre aquests temes i de veure que, en general. I'evaporacio ha suposat majors dificul. tats de comprensi $\delta$ als nostres alumnes que la condensacio. Aquest és un cas en què els alumnes no enfoquen correctament Ia reversibilitat, curiosament un dels requisits estudiats per Plaget quan estudia $1^{+}$accés dels nens o joves al seu estadi concret o formal.

- El rendiment depèr dels factors de disseny del quiestionari que s'han considerat; el context, el concepte o macrotema de les quiestions, $\mathrm{i}$ la intenci 6 de cada quiestió.

- També resulta funcío d'algunes variables sòcio-economiques i culturails que emmarquen la vida dels nostres alumnes: disposar d'un facil accés a llibres i a altres «béns» de tipus cultural, la llengua utilitzada usualment a classe, tenir unes aficions lligades a la natura i la seva conjuncí amb el fet de viure a zones metropolitanes o no, assistir a un centre o a un altre i que aquest sigui privat o públic (entre els 7 amb els quals hem treballat).

Finalment s'inclouen consideracions que poden ser d'utilitat als docents de Secundària, que tracten aquest tema dels canvis d'estat, i unes altres d'interès general. 